\title{
Adaptação das versões completa e breve da Escala de Relação Coparental (ERC) em uma amostra comunitária de pais e mães portugueses
}

\author{
Pedro Alexandre Costa ${ }^{1}$, Inês Queiroz Garcia' ${ }^{1}$, Fiona Tasker ${ }^{2}$ \& Isabel Leal ${ }^{1}$ \\ ${ }^{1}$ William James Center for Research, ISPA - Instituto Universitário \\ ${ }^{2}$ Birkbeck, University of London
}

\begin{abstract}
Resumo: A Escala de Relação Coparental (ERC) é uma das medidas mais utilizadas para avaliar a relação de coparentalidade. Pretendeu-se avaliar as propriedades psicométricas das versões completa e breve da ERC numa amostra comunitária de 779 pais e mães portugueses, com idades entre os 23 e os 65 anos ( $M$ = 42.73; $D P=5.27$ ). As análises fatoriais confirmatórias forneceram evidências de um bom ajustamento dos dados, fiabilidade interna e validade convergente, ainda que em ambas as versões tenha sido eliminada a dimensão Divisão de tarefas parentais. Quando à validade discriminante, duas das sete dimensões - Acordo nas práticas parentais e Suporte coparental - não evidenciaram validade discriminante. A validade concorrente também foi testada e confirmada através das correlações entre das dimensões da ERC e as dimensões da RDAS (ajustamento diádico) e do SDQ (ajustamento infantil).
\end{abstract}

\section{Palavras-chave: Coparentalidade; validação psicométrica; relação conjugal; ajustamento diádico.}

Adaptation of the complete and brief versions of the Coparenting Relationship Scale in a community sample of Portuguese fathers and mothers. The Coparenting Relationship Scale (ERC) is one of the most used measures to evaluate coparenting relationship. The aim of this study was to evaluate the psychometric properties of the complete and brief versions of the ERC in a community sample of 779 Portuguese fathers and mothers, aged between 23 and 65 years $(M=42.73, S D=5.27)$. Confirmatory factor analyzes provided evidence of good model fit, internal reliability, and convergent validity, although in both versions the Division of labor dimension was eliminated. Regarding discriminant validity, two out of the seven dimensions - Coparenting agreement and Coparenting support - did not show evidence of discriminant validity. Concurrent validity was also tested and confirmed through the correlations between the dimensions of the ERC and the dimensions of the RDAS (dyadic adjustment) and the SDQ (child adjustment).

\section{Keywords: Coparenting; psychometric validation; marital relationship; dyadic adjustment.}

Nos últimos anos, o conceito de coparentalidade tem assumido um valor preditivo do ajustamento psicológico da criança e dos pais/mães, bem como da compreensão do funcionamento familiar como um todo (Dorsey, Forehand, \& Brody, 2007; Feinberg, Brown, \& Kan, 2012; Lamela, Figueiredo, Bastos, \& Feinberg, 2015; Teubert \& Pinquart, 2010). Coparentalidade é definida como o conjunto de interações entre as figuras parentais (pais/mães) que dizem respeito ao desempenho das funções parentais como educação, formação, e tomada de decisões sobre a vida dos/as filhos/as (Feinberg, 2003; McConnell \& Kerig, 2002). Considerando que é necessária satisfação na relação de coparentalidade para o bom desenvolvimento da criança, assim como a escassez de instrumentos para avaliar este conceito, este estudo teve como objetivo a adaptação e avaliação das qualidades psicométricas da Escala de Relação Coparental (ERC; Coparenting Relationship Scale; Feinberg et al., 2012) para o contexto português, nas duas versões disponíveis no original; a versão completa e a versão breve.

As fundamentações e os modelos teóricos de coparentalidade estão inerentemente associados à teoria estrutural dos sistemas familiares (Altenburger, Schoppe-Sullivan, Lang, Bower, \& Kamp Dush, 2014; Minuchin, 1974), sendo que este conceito psicológico surgiu no contexto do estudo das relações familiares face ao divórcio (Frizzo, Kreutz, Schmidt, Piccinini, \& Bosa, 2005). Inserida no modelo teórico supramencionado, a coparentalidade, ou relação coparental, refere-se à interação das figuras parentais na partilha da responsabilidade na educação e na prestação de cuidados instrumentais e emocionais à

\footnotetext{
${ }^{1}$ Morada de correspondência: Pedro Alexandre Costa, William James Center for Research, ISPA - Instituto Universitário, Rua Jardim do Tabaco, 34, 1149-041 Lisboa, Portugal. E-mail: pcosta@ispa.pt. Este trabalho foi apoiado pela Fundação para a Ciência e a Tecnologia através de uma bolsa individual com a referência SFRH/BPD/99752/2014, atribuída ao primeiro autor.
} 
criança (Feinberg, 2003; Hohmann-Marriott, 2011; Lamela, Costa, \& Figueiredo, 2010; Margolin, Gordis, \& John, 2001; McHale, Kuersten-Hogan, \& Rao, 2004).

As concetualizações mais recentes da coparentalidade abordam o subsistema com uma maior extensão, considerando-o como universal e autónomo da estrutura familiar (Feinberg, 2003; Van Egeren \& Hawkins, 2004). No entanto, McHale (1995) sublinha que a coparentalidade - subsistema executivo desempenha uma função primordial no contexto familiar, influenciando o funcionamento interno de cada indivíduo, as relações entre os pais/mães, e entre estes e a criança (Favez, Tissot, Frascarolo, Stiefel, \& Despland, 2016; Reader, Teti, \& Cleveland, 2017). A relação coparental, apesar de estar associada à relação conjugal, difere da mesma pois é concetualizada através de uma abordagem triádica (pai(s)/mãe(s)/criança), que não inclui os aspetos financeiros, românticos, sexuais e emocionais do relacionamento conjugal, e não se reduz aos estilos e práticas educativas das figuras parentais em relação aos/às filhos/as (Feinberg, 2003; Holland \& McElwain, 2013; Jia \& Schoppe-Sullivan, 2011).

Neste sentido, são diversos os autores que ao longo dos anos apresentaram propostas teóricas por forma a contribuírem para uma melhor concetualização no estudo da coparentalidade (Belsky, Crnic, \& Gable, 1995; Margolin et al., 2001; McHale, 1995; Van Egeren \& Hawkins, 2004). No entanto, foi através do Modelo da Estrutura Interna e Contexto Ecológico da Coparentalidade desenvolvido por Mark Feinberg (2003) que o conceito de coparentalidade ganhou maior relevância empírica. Feinberg (2002, 2003) propôs que a coparentalidade positiva está relacionada com a competência parental percebida pelas figuras parentais (e.g., saúde mental e/ou expetativas acerca dos papéis parentais), com o comportamento da criança, com uma parentalidade democrático-recíproca (e.g., repertório comportamental das figuras parentais enquanto cônjuges, em famílias nucleares) e pelo ambiente extrafamiliar (e.g., crise económica e/ou suporte social). Feinberg (2003; Feinberg et al., 2012) propôs então um quadro concetual de coparentalidade que assenta em quatro dimensões: (a) Acordo nas práticas parentais, onde é compreendido o grau de concordância (ou discordância) entre as figuras parentais sobre aspetos relacionados com a criança (e.g., princípios morais, disciplina, formas de prestação de cuidados, decisões sobre as necessidades emocionais e a sua segurança). 0 facto de as figuras parentais discordarem pode resultar em prejuízo - críticas e hostilidade - no âmbito do funcionamento familiar, podendo afetar a consistência das estratégias educativas usadas pelos pais/mães. Apesar disso, as figuras parentais quando "concordam em discordar" conseguem manter níveis elevados de apoio mútuo coparental (Feinberg, 2002); (b) Divisão do trabalho relacionado com a criança, que compreende a divisão de tarefas e responsabilidades inerentes ao cuidado e assistência à criança, às tarefas domésticas e responsabilidades financeiras, legais e médicas, relacionadas com a criança; (c) Suporte/sabotagem do papel coparental, que se relaciona com o grau de suporte mútuo existente entre as figuras parentais, manifestado através da validação das competências de um deles perante os contributos do papel parental do outro, e, por outro, evidente por padrões de hostilidade e de depreciação perante o parceiro a fim de gerar culpa no outro (Feinberg, 2003); e, (d) Gestão conjunta das relações familiares, que surge como uma poderosa responsabilidade do subsistema executivo, onde são compreendidos três aspetos: conflitos, coligações, e equilíbrio da regulação emocional familiar. 0 grau em que a criança é exposta a alianças e/ou conflitos interparentais surge como um dos aspetos fundamentais desta responsabilidade, devendo ser procurado o equilíbrio na interação entre pais/mães e a criança (Feinberg, 2003).

No decorrer das concetualizações em torno do construto da coparentalidade, e em particular do ponto de vista metodológico, foram vários os instrumentos desenvolvidos com o intuito de avaliar a coparentalidade, desde medidas observacionais (e.g., Coparenting and Family Rating System; McHale, Kuersten-Hogan, \& Lauretti, 2001) a medidas de autorrelato (e.g., Parental Alliance Inventory; Abidin \& Brunner, 1995). Contudo, entre os instrumentos de autorrelato de avaliação da coparentalidade, a Escala de Relação Coparental desenvolvida por Feinberg et al. (2012) com base nas dimensões do modelo ecológico da coparentalidade, tem sido das mais utilizadas e investigadas.

Feinberg et al. (2012) desenvolveram sete dimensões com base nas quatro dimensões do modelo teórico, tendo sido feita uma divisão a priori: (1) Acordo nas práticas parentais; (2) Divisão de tarefas parentais; suporte/sabotagem do papel coparental foi subdividida em três dimensões: (3) Suporte coparental, (4) Sabotagem, e (5) Aprovação parental; gestão conjunta das relações familiares foi renomeada para (6) Exposição ao conflito; e, por fim, uma sétima subescala distinta das dimensões da coparentalidade de Feinberg (2003) foi acrescentada com o intuito de determinar o grau da intimidade e a força da relação coparental, (7) Proximidade coparental. No que se refere à sua aplicabilidade, a ERC tem sido amplamente utilizada em estudos que avaliam a coparentalidade em diversos contextos, como o apoio coparental na amamentação (Abbass-Dick, Stern, Nelson, Watson, \& Dennis, 2015), a observação da coparentalidade e do temperamento da criança para o seu ajustamento socio-emocional (Altenburger, Lang, Schoppe-Sullivan, Kamp Dush, \& Johnson, 2017), o desenvolvimento de uma medida de 
compreensão da gatekeeping maternal para pais/mães de crianças em idade pré-escolar (Puhlman \& Pasley, 2017), bem como na avaliação da coparentalidade durante a gravidez (Altenburger et al., 2014; Favez, Frascarolo, Lavanchy Scaiola, \& Corboz-Warnery, 2013). Para além da versão completa da ERC (35 itens), Feinberg et al. (2012) desenvolveram uma versão breve retendo os dois itens concetualmente e empiricamente mais representativos de cada subescala. A versão breve permite que a coparentalidade possa ser avaliada em outros contextos que não clínicos onde o tempo é mais limitado, e de forma a que não sejam sacrificadas outras medidas de avaliação nem a qualidade dos dados obtidos (Feinberg et al., 2012).

No presente estudo, compreende-se então o construto da coparentalidade a partir do modelo proposto por Feinberg (2003). A relação coparental - com um papel central na família nuclear - está associada aos cuidados e deveres em relação à criança que ambas as figuras parentais têm entre si na díade coparental, sem envolver dimensões da conjugalidade. Apesar de serem diversas as investigações no âmbito da coparentalidade e do modelo proposto por Feinberg (2003; Feinberg et al., 2012) surgir como um ponto de referência no estudo da coparentalidade (Lamela et al., 2010; Schoppe-Sullivan \& Mangelsdorf, 2013), o uso da ERC ainda é escasso em Portugal.

Em contexto português, Lamela e colaboradores utilizaram a ERC em alguns estudos, nomeadamente na adaptação e validação da versão completa da ERC numa amostra de mães portuguesas (Lamela, Morais, \& Jongenelen, 2018) e na versão breve da ERC numa amostra de pais divorciados portugueses (Lamela et al., 2015). Também Pinto, Figueiredo, e Feinberg (2019) criaram uma versão da ERC para pais durante a gravidez - Father's Prenatal version (Pinto, Figueiredo, \& Feinberg, 2019). No entanto, nenhum deles materializou a adaptação de ambas as versões (completa ou breve) da Escala de Relação Coparental de Feinberg et al. (2012) em pais ou mães portugueses. 0 presente estudo pretende assim examinar as propriedades psicométricas de ambas as versões da ERC, permitindo posicionar a ERC em termos teóricos e empíricos no contexto do estudo da coparentalidade e promovendo a adequação cultural da ERC na população portuguesa.

\section{MÉTODO}

\section{Participantes}

Um total de 779 questionários foram preenchidos por pais/mães, e apenas um pai (30\%) ou uma mãe $(70 \%)$ em cada família, como solicitado no consentimento informado. A idade dos pais/mães variou entre os 23 e os 65 anos $(M=42.73 ; D P=5.27)$, com cerca de $80 \%$ reportando estar num relacionamento legalmente reconhecido (casamento ou união de facto), e apenas cerca de $14 \%$ não se encontrando no momento do estudo num relacionamento com outro pai/mãe dos filhos. A maioria dos pais/mães participantes reportaram elevado nível de escolaridade, habitar em localidade urbana, trabalhar a tempo inteiro e manter uma ocupação profissional ou de gestão/técnica. No que diz respeito aos/às filhos/as dos participantes, a idade variou entre os 6 e os 18 anos de idade $(M=12.09 ; D P=3.75)$. 0 número total de filhos/as variou entre um e sete, tendo sido calculada uma média de dois filhos/as por participante. Finalmente, o número de pessoas no agregado familiar variou entre uma e nove, tendo sido obtida uma média de quatro pessoas por agregado (Tabela 1).

\section{Instrumentos}

Todos os participantes providenciaram informações sobre os/as companheiros (e.g., idade, género, nível de escolaridade, ocupação) e informação relativa ao relacionamento (e.g., tempo da relação, satisfação com a relação). Os participantes também completaram medidas de ajustamento diádico do casal, deteção de problemas comportamentais e emocionais e avaliação da sintomatologia de humor nos/as filhos/as e, por fim, a versão portuguesa da Escala de Relação Coparental.

Revised Dyadic Adjustment Scale (RDAS) - Escala de Ajustamento Diádico Revista. Busby, Christensen, Crane, and Larson (1995) propuseram uma versão breve da Escala de Ajustamento Diádico (32 itens; Spanier, 1976), com o propósito de avaliar a satisfação relacional. A RDAS é composta por 14 itens divididos em três dimensões de ajustamento diádico: (a) Consenso: 6 itens (0 = "Sempre de acordo" a 5 = "Sempre em desacordo"); (b) Satisfação: 4 itens (0 = "Sempre" a 5 = "Nunca"); e (c) Coesão: 4 itens $(0=$ "Nunca" a 5 = "Frequentemente"). Existem evidências psicométricas que demonstram a boa aplicabilidade da RDAS em diferentes contextos, nomeadamente em Portugal (Costa, Pereira, \& Leal, 2011). Todas as dimensões revelaram uma fiabilidade moderada a elevada, Consenso ( $\alpha=.79$ ), Satisfação $(\alpha=.82)$ e Coesão $(\alpha=.81)$. Resultados mais elevados nas três dimensões refletem um maior ajustamento diádico. 
Tabela 1. Caracterização dos participantes

\begin{tabular}{|c|c|}
\hline & $n(\%)$ \\
\hline \multicolumn{2}{|l|}{ Tipo de relacionamento $*$} \\
\hline Casado(a) & $525(67.4)$ \\
\hline União de facto & $96(12.3)$ \\
\hline Coabitação (sem vínculo legal) & $38(4.9)$ \\
\hline Sem coabitação (sem vínculo legal) & $20(2.6)$ \\
\hline Solteiro(a) & $36(4.6)$ \\
\hline Divorciado(a)/Separado(a) & $63(8.1)$ \\
\hline Viúvo(a) & $1(0.1)$ \\
\hline \multicolumn{2}{|l|}{ Local de habitação } \\
\hline Urbano/Grande cidade & $332(42.7)$ \\
\hline Periferia urbana & $222(28.5)$ \\
\hline Semiurbano/Cidade pequena & $107(13.8)$ \\
\hline Vila & $68(8.7)$ \\
\hline Rural & $49(6.3)$ \\
\hline \multicolumn{2}{|l|}{ Nível de escolaridade } \\
\hline Ensino médio & $25(3.2)$ \\
\hline Ensino secundário & $119(15.3)$ \\
\hline Ensino pós-secundário (especialização tecnológica) & $33(4.2)$ \\
\hline Pós-graduação & $130(16.7)$ \\
\hline Curso superior & $472(60.6)$ \\
\hline \multicolumn{2}{|l|}{ Situação profissional } \\
\hline Emprego a tempo inteiro & $654(84.2)$ \\
\hline Emprego a tempo parcial & $29(3.7)$ \\
\hline Por conta própria/independente & $19(2.4)$ \\
\hline Desemprego & $53(6.8)$ \\
\hline Reforma & $6(0.8)$ \\
\hline Outra & $16(2.1)$ \\
\hline \multicolumn{2}{|l|}{ Ocupação } \\
\hline Profissional & $373(49.5)$ \\
\hline Gestão/Técnica & $262(34.8)$ \\
\hline Especializada não manual & $39(5.2)$ \\
\hline Especializada manual & $12(1.6)$ \\
\hline Parcialmente especializada & $13(1.7)$ \\
\hline Não especializada & $19(2.5)$ \\
\hline Outra & $35(4.6)$ \\
\hline \multicolumn{2}{|l|}{ Rendimento anual do agregado familiar } \\
\hline Até €7.000 & $54(7.1)$ \\
\hline Entre $€ 7.001$ e $€ 20.000$ & $204(26.9)$ \\
\hline Entre $€ 20.001$ e $€ 40.000$ & $267(35.2)$ \\
\hline Entre €40.001 e €80.000 & $183(24.1)$ \\
\hline Mais de $€ 80.000$ & $51(6.7)$ \\
\hline
\end{tabular}

Nota. *Não obstante alguns pais/mães reportarem não estar numa relação no momento, todos os participantes reportaram partilhar a coparentalidade dos seus filhos.

Strengths and Difficulties Questionnaire (SDQ) - Questionário de Capacidades e Dificuldades. Os participantes do estudo completaram a versão de pais/mães (crianças entre os 4 e os 17 anos) do Questionário de Capacidades e Dificuldades (versão portuguesa e versão original, respetivamente: Fleitlich, Loureiro, Fonseca, \& Gaspar, 2005; Goodman, 1997). Apesar de serem propostas cinco 
subescalas compostas por cinco itens cada, estudos recentes sugerem que a versão de três subescalas é mais robusta e mais indicada para amostras comunitárias com baixo risco de problemáticas (Costa, Tasker, Ramos, \& Leal, 2020; Goodman, Lamping, \& Ploubidis, 2010). A versão de três subescalas é composta por (a) Problemas de Internalização; (b) Problemas de Externalização; e (c) Comportamentos Pró-sociais. É pedido aos/as pais/mães que indiquem em que medida observaram cada um dos comportamentos no seu filho/a nos últimos seis meses, numa escala tipo Likert de 3 pontos $(0=$ "Não é verdade", 1 = "É um pouco verdade", 2 = "É muito verdade"). O SDQ é amplamente utilizado pela sua sensibilidade na deteção de problemas comportamentais e emocionais, existindo evidência da sua validade em comparação com outras medidas bem estabelecidas, como a Child Behavior CheckList (CBCL; Klasen et al., 2000; Rothenberger \& Woerner, 2004; Warnick, Bracken, \& Kasl, 2008). A consistência interna demonstrou ser moderada para as dimensões Problemas de Internalização $(\alpha=.74)$ e Problemas de Externalização $(\alpha=.78)$, e aceitável para a dimensão Comportamentos Pró-sociais $(\alpha=.69)$ no presente estudo.

Coparenting Relationship Scale (CRS no original) - Escala de Relação Coparental (ERC). Feinberg et al. (2012) desenvolveram uma medida de autorrelato para avaliar a coparentalidade com base no Modelo Ecológico de Feinberg (2003). Esta permite analisar a perceção que o pai ou a mãe têm do apoio do/a parceiro/a e da coordenação de ambos, na educação do/a seu/sua filho/a. Assim como na versão original, a versão portuguesa da ERC (Lamela et al., 2018) é composta por 35 itens cujas respostas variam numa escala de Likert de 7 pontos ( 0 = "Não é verdadeiro sobre nós"; 6 = "Muito verdadeiro sobre nós"). É composta pelas sete subescalas medidas nas cinco dimensões da coparentalidade: Acordo nas práticas parentais (4 itens; e.g., "Eu e o/a meu/minha parceiro/a temos os mesmos objetivos para o/a nosso/a filho/a"); Proximidade coparental (5 itens; e.g., "Eu sinto-me próximo/a do/a meu/minha parceiro/a quando o/a vejo a brincar com o/a nosso/a filho/a"); Exposição ao conflito (5 itens; e.g., "Um/a ou ambos/as dizem coisas cruéis ou ofensivas um/a ao outro/a à frente do/a seu/sua filho/a?"); Suporte coparental (6 itens; e.g., "Quando chego ao meu limite como pai/mãe, o/a meu/minha parceiro/a dá-me o apoio extra que eu preciso"); Sabotagem (6 itens; e.g., "0/a meu/minha parceiro/a por vezes faz piadas ou comentários sarcásticos sobre mim como pai/mãe"); Aprovação parental (7 itens; e.g., "Eu acho que o/a meu/minha parceiro/a é um/a bom/a pai/mãe”); e, Divisão de tarefas (2 itens; e.g., "0/a meu/minha parceiro/a gosta de brincar com o/a nosso/filho/a e depois deixa a desarrumação para mim"). A versão breve da ERC é composta por 14 itens $(1,2,4,5,6,9,16,20,22,24,25,27,33$ e 34), que foram selecionados das sete subescalas (dois itens por subescala), como proposto por Feinberg (2003). Na presente investigação os índices de consistência interna são apresentados na secção Resultados.

\section{Procedimentos}

Os participantes (pais/mães) foram recrutados através de um email de divulgação do estudo "Parentalidade: Capacidades e Dificuldades" dirigido a escolas e associações de pais em Portugal, que descrevia os objetivos do estudo e as instruções de participação. Foram também facultados os contatos do investigador responsável (primeiro autor) para esclarecimento de qualquer questão sobre o estudo. 0 questionário foi alojado online na plataforma Qualtrics. De acordo com a Declaração de Helsínquia, todos os participantes tiveram a oportunidade de esclarecer dúvidas sobre os objetivos e/ou procedimentos do estudo por meio de e-mail, bem como foi garantido o anonimato e a confidencialidade antes da aceitação em participar no estudo. Nenhuma compensação foi oferecida aos participantes. Foi obtido o consentimento informado por parte de todos os participantes neste estudo. 0 parecer positivo da Comissão de Ética do ISPA - Instituto Universitário foi obtido antes do início do recrutamento de participantes.

Análise Estatística. As análises estatísticas foram realizadas utilizando o software IBM SPSS Statistics (v. 25) e o programa estatístico AMOS (v. 25). A sensibilidade dos itens foi avaliada através da análise da assimetria (Sk) e da curtose (Ku), com valores absolutos inferiores a três e a sete, respetivamente, denotando ausência de violação grave do pressuposto de normalidade (Kline, 2000). Os modelos hipotéticos de sete fatores e de um fator para a ERC foram testados por meio da análise fatorial confirmatória (AFC). Com base na significância estatística das estimativas de parâmetros, apenas itens com um nível de probabilidade inferior a .05 , pesos de regressão estandardizados de .40 ou superior, e cargas fatoriais estandardizadas maiores que .15 foram retidos (Marôco, 2014).

0 ajustamento global do modelo foi avaliado usando o rácio Qui-Quadrado / graus de liberdade $\left(\chi^{2} / \mathrm{gl}\right)$, Comparative Fit Index (CFI), Goodness of Fit Index (GFI) e Root Mean Square Error of Approximation (RMSEA). 0 modelo foi considerado com um ajustamento aceitável ("sofrível") aos dados 
se $\chi^{2} / \mathrm{gl}$ foi inferior a 5, CFI e GFI foi superior a .80, e RMSEA foi inferior a .10, e com um ajustamento bom aos dados se $\chi^{2} / \mathrm{gl}$ foi inferior a 2, CFI e GFI foi superior a .90, e RMSEA foi inferior a .05 (Marôco, 2014).

A validade convergente foi avaliada através do cálculo da Variância Extraída da Média (VEM; Fornell \& Larcker, 1981), uma medida da quantidade de variância que é capturada pelo fator latente em relação à quantidade de variância que pode ser atribuída ao erro de medição. Sempre que a VEM for maior que .50, a validade convergente pode ser considerada adequada (Fornell \& Larcker, 1981; Hair, Black, Babin, \& Anderson, 2009). A validade discriminante foi explorada calculando a correlação ao quadrado dos interfatores com a VEM de cada fator. Para demonstrar a validade discriminante dos fatores, a VEM dos fatores individuais deve ser maior que a correlação ao quadrado entre os fatores (Marôco, 2014).

A consistência interna (alfa de Cronbach) e a Fiabilidade Compósita (FC) foram calculadas para cada uma das dimensões da ERC (Fornell \& Larcker, 1981; Hair et al., 2009). 0 alfa de Cronbach e a FC foram considerados como tendo boa fiabilidade se fossem maiores que .80 (Kline, 2000). Finalmente, a validade concorrente foi avaliada pelo cálculo dos coeficientes de correlação de Pearson entre as dimensões da ERC e medidas de critério (Cohen, 1988).

\section{RESULTADOS}

\section{Validade de Construto}

Foram realizadas Análises Fatoriais Confirmatórias (AFC) para testar as estruturas fatoriais da versão completa (35 itens) e da versão breve (14 itens) da ERC. A normalidade multivariada foi confirmada para todos os itens de ambas as versões, não sendo identificados problemas significativos de assimetria $(|\mathrm{Sk}|<$ 3) ou de curtose $(|\mathrm{Ku}|<7)$.

Análise fatorial confirmatória da versão completa (35 itens) da ERC. 0 modelo original de sete fatores relativo à versão completa da ERC foi testado, tendo-se encontrado um ajustamento inicial "sofrível", $\chi^{2} / \mathrm{gl}=4.36 ; \mathrm{CFI}=.89 ; \mathrm{GFI}=.84$, RMSEA = .07; $p<.001 ; 90 \% \mathrm{CI}[.063-.068]$. Com base na análise dos pesos fatoriais, o item 13 ("O/a meu/minha parceiro/a não confia nas minhas capacidades como pai/mãe") e o item 28 ("O stress de parentalidade levou a que eu e o/a meu/minha parceiro/a nos tornássemos distantes") apresentaram pesos fatoriais estandardizados abaixo do aceitável $(\lambda=.26$ e $\lambda=$ .36, respetivamente). Além disso, o valor das cargas fatoriais estandardizadas do item 5 ("O/a meu/minha parceiro/a gosta de brincar com o/a nosso/filho/a e depois deixa a desarrumação para mim") e do item 20 ("O/a meu/minha parceiro/a não assume a sua cota parte das tarefas parentais") estavam abaixo do recomendado $\left(r^{2}=-.01\right.$ e $r^{2}=-.04$, respetivamente). Estes quatro itens foram excluídos, sendo por consequência eliminado o fator Divisão de tarefas, que era composto apenas pelos itens 5 e 20, e o modelo foi reajustado, revelando uma melhoria, $\chi^{2} / \mathrm{gl}=4.69 ; \mathrm{CFI}=.90 ; \mathrm{GFI}=.85$, RMSEA $=.07 ; p<.001$; 90\% CI [.066-.072].

Os índices de modificação apontaram para uma correlação elevada dos erros dos seguintes pares de itens: 10-27, 26-27, 10-25, 25-27 e 9-15. Após a inserção das correlações, a nova estrutura fatorial de seis fatores foi testada tendo o modelo apresentado um ajustamento aceitável, $\chi^{2} / \mathrm{gl}=3.83$; CFI = .93; GFI $=.88$, RMSEA $=.06 ; p<.001 ; 90 \%$ CI [.057-.064]. A Figura 1 apresenta os pesos fatoriais estandardizados $\mathrm{e}$ a fiabilidade dos itens individuais da versão completa de seis fatores (31 itens) da ERC.

Análise fatorial confirmatória da versão breve (14 itens) da ERC. 0 modelo de um único fator relativo à versão breve original da ERC foi testado, tendo-se encontrado um ajustamento inicial não aceitável, $\chi^{2} / \mathrm{gl}=18.13 ; \mathrm{CFI}=.76$; GFI $=.79$, RMSEA $=.15 ; p<.001 ; 90 \% \mathrm{CI}[.142-.155]$. Com base na análise dos pesos fatoriais, o item 5 e o item 20 (dimensão Divisão de tarefas) apresentaram pesos fatoriais estandardizados $(\lambda=.22$ e $\lambda=-.40$, respetivamente) abaixo do aceitável sendo por isso eliminados. 0 item 33 ("Discute sobre a sua relação ou problemas conjugais não relacionados com o/a seu/sua filho/a, na presença dele/a?"; correspondente ao fator Exposição ao conflito) foi também eliminado por apresentar um peso fatorial estandardizado e um valor da carga fatorial estandardizada abaixo do aceitável $\left(\lambda=.18 ; r^{2}=.03\right)$. Após a eliminação dos três itens, o modelo foi reajustado revelando uma melhoria, $\chi^{2} / \mathrm{gl}=17.86$; CFI = .84; GFI = .83, RMSEA $=.15 ; p<.001 ; 90 \% \mathrm{CI}$ [.138-.156]. Os índices de modificação apontaram para uma correlação elevada dos erros dos seguintes pares de itens: 25-27, 1622, e 1-4. Após a inserção das correlações, a nova estrutura fatorial apresentou um ajustamento aceitável, $\chi^{2} / \mathrm{gl}=8.30 ; \mathrm{CFI}=.94 ; \mathrm{GFI}=.92 ; \mathrm{RMSEA}=.09 ; p<.001 ; 90 \% \mathrm{CI}[.087-.106]$. A Figura 2 apresenta os pesos fatoriais estandardizados e a fiabilidade dos itens individuais da versão breve da ERC, que passou de 14 itens para uma versão de 11 itens ajustada à amostra deste estudo. 


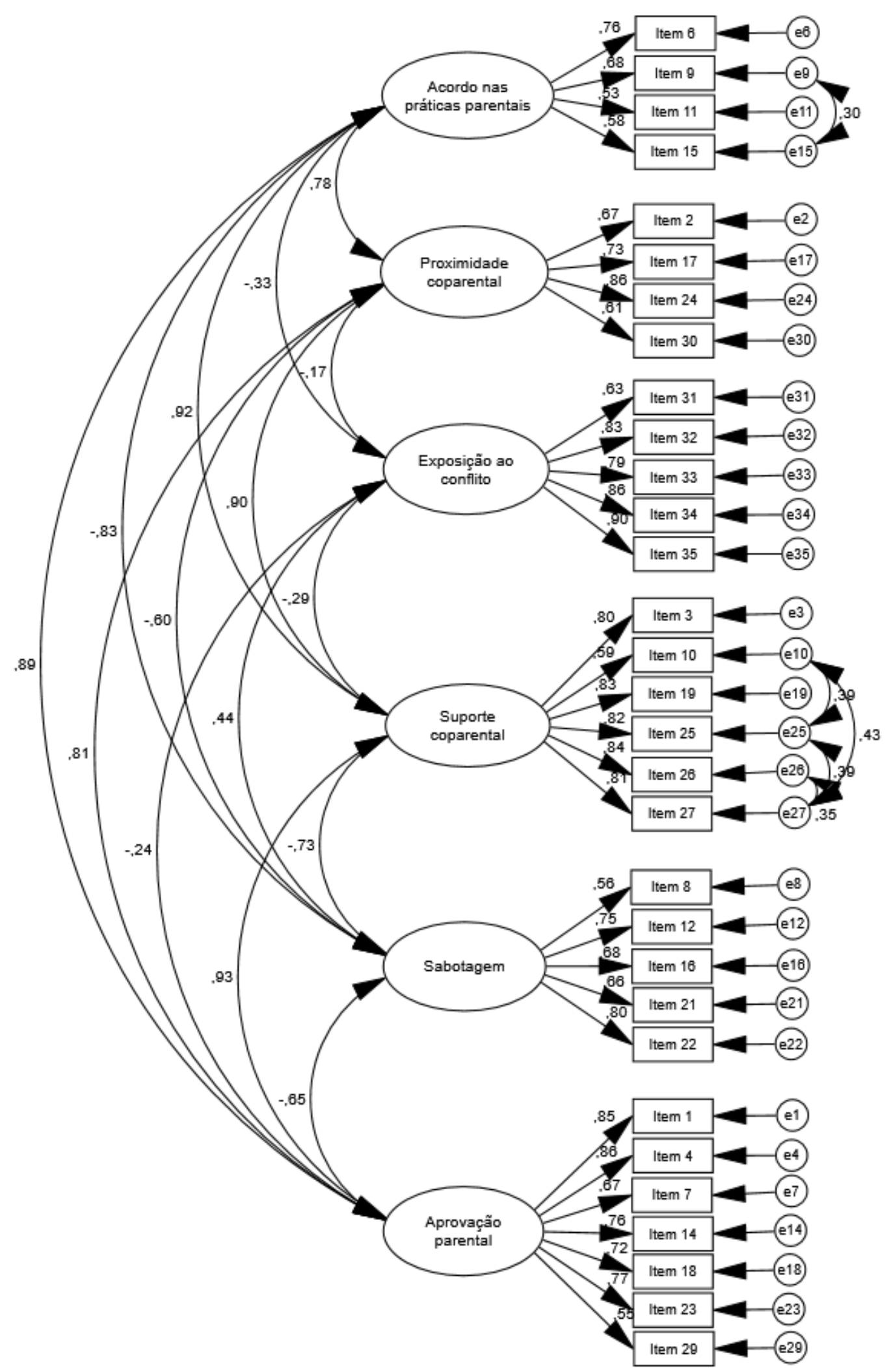

Figura 1. Análise Fatorial Confirmatória da versão completa da Escala de Relação Coparental. 


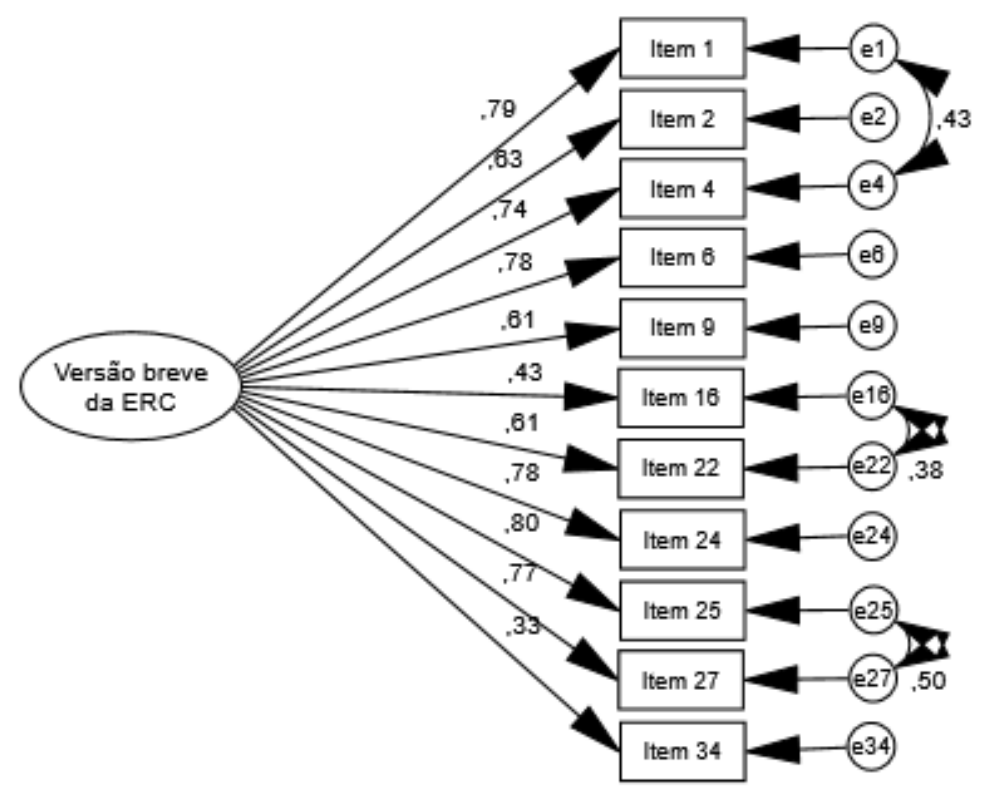

Figura 2. Análise Fatorial Confirmatória da versão breve da Escala de Relação Coparental.

As análises estatísticas subsequentes para testar a fiabilidade, validade discriminante, convergente e de critério foram realizadas com base nesta distribuição de itens pelos seis fatores (versão completa) e na versão unifatorial (versão breve) da ERC.

Fiabilidade. A consistência interna da versão completa de seis fatores (31 itens) e da versão breve (11 itens) está descrita na Tabela 2 . Todas as dimensões evidenciaram uma fiabilidade aceitável a boa $(.77<\alpha$ $<.92$ e $.74<F C<.97)$. 0 modelo da versão breve evidenciou uma fiabilidade aceitável $(\alpha=.73)$, apesar do valor de fiabilidade compósita ser considerado bom $(F C=.90)$.

Tabela 2. Alfas de Cronbach $(\alpha)$, Fiabilidade Compósita (FC) e Variância Extraída da Média (VEM) das duas versões da ERC

\begin{tabular}{|c|c|c|c|c|c|c|c|}
\hline & \multicolumn{6}{|c|}{ Versão completa } & \multirow{2}{*}{$\begin{array}{c}\begin{array}{c}\text { Versão } \\
\text { breve }\end{array} \\
\text { Total }\end{array}$} \\
\hline & $\begin{array}{c}\text { Acordo nas } \\
\text { práticas } \\
\text { parentais }\end{array}$ & $\begin{array}{l}\text { Proximidade } \\
\text { coparental }\end{array}$ & $\begin{array}{l}\text { Exposição ao } \\
\text { conflito }\end{array}$ & $\begin{array}{c}\text { Suporte } \\
\text { coparental }\end{array}$ & Sabotagem & $\begin{array}{c}\text { Aprovação } \\
\text { parental }\end{array}$ & \\
\hline$\alpha$ & .77 & .81 & .89 & .92 & .80 & .89 & .73 \\
\hline FC & .74 & .81 & .90 & .91 & .82 & .90 & .90 \\
\hline VEM & .42 & .53 & .65 & .62 & .48 & .56 & .46 \\
\hline
\end{tabular}

Validade convergente. A validade convergente da ERC foi testada através do cálculo da VEM. As dimensões da ERC apresentaram maioritariamente valores aceitáveis (i.e., > .50) e variando entre .48 para a dimensão Sabotagem e .65 para a dimensão Exposição ao conflito, excetuando-se a dimensão Acordo nas práticas parentais que apresentou um valor de VEM de .42 (Tabela 2).

Validade discriminante. A validade discriminante para as dimensões da ERC está descrita na Tabela 3. Das quinze possíveis comparações, apenas setes apresentaram validade discriminante, destacando-se duas dimensões em particular: Acordo nas práticas parentais e Suporte coparental, que partilham mais variância com outras dimensões de coparentalidade do que o aceitável, e por isso sugerindo não serem indicadores empiricamente válidos dos construtos que teoricamente se propõem a avaliar (Tabela 3). 
Tabela 3. Validade discriminante das dimensões da versão completa de seis fatores da ERC

\begin{tabular}{ll}
\hline Associações entre fatores & $\boldsymbol{r}^{2}$ \\
\hline Acordo nas práticas parentais $\leftrightarrow$ Proximidade coparental & .61 \\
Acordo nas práticas parentais $\leftrightarrow$ Exposição ao conflito & $.11^{*}$ \\
Acordo nas práticas parentais $\leftrightarrow$ Suporte coparental & .84 \\
Acordo nas práticas parentais $\leftrightarrow$ Sabotagem & .68 \\
Acordo nas práticas parentais $\leftrightarrow$ Aprovação parental & .80 \\
Proximidade coparental $\leftrightarrow$ Exposição ao conflito & $.03^{*}$ \\
Proximidade coparental $\leftrightarrow$ Sabotagem & $.36^{*}$ \\
Proximidade coparental $\leftrightarrow$ Aprovação parental & .66 \\
Exposição ao conflito $\leftrightarrow$ Suporte coparental & $.08^{*}$ \\
Exposição ao conflito $\leftrightarrow$ Sabotagem & $.20^{*}$ \\
Exposição ao conflito $\leftrightarrow$ Aprovação parental & $.06^{*}$ \\
Suporte coparental $\leftrightarrow$ Sabotagem & .54 \\
Suporte coparental $\leftrightarrow$ Aprovação parental & .86 \\
Suporte coparental $\leftrightarrow$ Proximidade coparental & .81 \\
Sabotagem $\leftrightarrow$ Aprovação parental & $.42^{*}$ \\
\hline
\end{tabular}

Nota. * Assinala evidência de validade discriminante.

\section{Validade de Critério}

Validade concorrente. Para avaliar a validade concorrente da ERC e das suas dimensões com as medidas de critério (ajustamento diádico e ajustamento infantil), foram calculadas correlações bivariadas entre todas as subescalas (Tabela 4). Todas as associações foram estatisticamente significativas e na direção prevista. As subescalas da RDAS evidenciaram correlações negativas moderadas a fracas $(-.60<r<-.24)$ com as dimensões Acordo nas práticas parentais, Exposição ao conflito e Sabotagem. De forma similar, as subescalas da RDAS evidenciaram correlações positivas moderadas a fracas $(.46<r<.24)$ com as dimensões Proximidade coparental, Suporte coparental, Aprovação parental e com a escala breve da ERC. As subescalas Problemas de Internalização e Problemas de Externalização do SQD evidenciaram correlações negativas fracas $(-.21<r<-.12)$ com as dimensões Proximidade coparental, Suporte coparental, Aprovação parental e com a escala breve da ERC. Por sua vez, as restantes dimensões da ECR evidenciaram correlações positivas fracas $(.23<r<.16)$ com as subescalas Problemas de Internalização e Problemas de Externalização do SDQ. Quanto à subescala Comportamentos Pró-sociais, as correlações foram fracas e significativas e na direção prevista.

Tabela 4. Validade concorrente entre a versão completa e a versão breve da ERC e medidas de critério

\begin{tabular}{lcccccc}
\hline & $\begin{array}{c}\text { Consenso Satisfação } \\
\text { (a) }\end{array}$ & $\mathbf{( a )}$ & $\begin{array}{c}\text { Coesão } \\
\text { (a) }\end{array}$ & $\begin{array}{c}\text { Problemas de } \\
\text { Internalização } \\
\text { (b) }\end{array}$ & $\begin{array}{c}\text { Problemas de Comportamentosação } \\
\text { (b) }\end{array}$ & $\begin{array}{c}\text { Pró-sociais } \\
\text { (b) }\end{array}$ \\
\hline Acordo nas práticas parentais & -.28 & -.28 & -.24 & .22 & .17 & -.11 \\
Proximidade coparental & .34 & .34 & .30 & -.18 & -.12 & .12 \\
Exposição ao conflito & -.48 & -.60 & -.35 & .19 & .21 & -.13 \\
Suporte coparental & .46 & .41 & .39 & -.21 & -.16 & .17 \\
Sabotagem & -.35 & -.36 & -.25 & .23 & .16 & -.13 \\
Aprovação parental & .28 & .31 & .24 & -.19 & -.15 & .10 \\
Versão breve & .37 & .35 & .33 & -.17 & -.14 & .13
\end{tabular}

Nota 1. (a) Subescalas que pertencem à Escala de Ajustamento Diádico Revista. (b) Subescalas que pertencem ao Questionário de Capacidades e Dificuldades.

Nota 2. Todas as correlações são significativas ao nível de .01. 


\section{DISCUSSÃO}

A Escala de Relação Coparental é uma das medidas mais comumente usadas para avaliar a qualidade da relação de coparentalidade, que se assume como um importante componente do funcionamento familiar como um todo, e com um valor preditivo relevante do ajustamento psicossocial das crianças (Dorsey et al., 2007; Feinberg, 2002; Feinberg et al., 2012). Contudo, esta é uma escala de medida ainda pouco avaliada no que diz respeito às suas qualidades psicométricas fora do contexto Norte Americano, onde foi desenvolvida. Assim, este estudo teve como objetivo avaliar as propriedades psicométricas da versão portuguesa da Escala de Relação Coparental (ERC) e desta forma contribuir para a sua adaptação para o contexto português. Os resultados globais evidenciaram confiabilidade e validades de construto e de critério para ambas as versões completa e breve da ERC, ainda que após a eliminação de alguns itens em cada uma das versões. Desta forma, este estudo sugere que as versões completa e breve de ERC são confiáveis para avaliar a relação de coparentalidade em pais/mães portugueses ainda que requeiram mais estudos que corroborem a sua estrutura definitiva.

No que diz respeito à versão completa da ERC, a análise fatorial confirmatória não suportou a estrutura original de sete fatores proposta pelos autores uma vez que os dois únicos itens que compõem a dimensão Divisão de tarefas parentais foram eliminados por evidenciarem baixos valores de carga fatorial. Este resultado tinha já sido evidenciado em um estudo com uma amostra de mães portuguesas (Lamela et al., 2018), tendo os autores argumentado que a divisão de tarefas parece ser concetualmente distante da coparentalidade. Mais, Lamela et al. (2018) e Feinberg et al. (2012) sugerem que a divisão de tarefas é culturalmente situada e com alta variabilidade entre países, não sendo por isso um construto basilar na avaliação da qualidade da coparentalidade. Também no estudo de validação inicial de Lamela et al. (2018), o item 28 ("O stress de parentalidade levou a que eu e o/a meu/minha parceiro/a nos tornássemos distantes") foi eliminado por não ter obtido um peso fatorial aceitável, ainda que os autores tenham eliminado outros dois itens (itens 6 e 7) que no presente estudo revelaram pesos fatoriais aceitáveis. A eliminação do item 28 no estudo de Lamela et al. (2018), assim como no presente estudo, parece indicar a sua fraca fiabilidade, sugerindo-se que este item não seja útil em amostras comunitárias e de baixo risco entre as quais os níveis de stress parental são baixos.

Para corrigir as correlações entre os erros dos itens (avaliados por meio de índices de modificação - Multiplicadores de Lagrange), foram assumidas quatro correlações para melhorar o ajustamento do modelo. Correlações de erros entre os itens 10-27, 26-27, 10-25, e 25-27 da dimensão Suporte coparental. Em particular, o item 27 parece especialmente problemático, cujo erro revelou uma correlação elevada com outros três erros de medida, representando uma forte comunalidade subjacente entre este item e os restantes três. Resultados semelhantes foram reportados no estudo de Lamela et al. (2018), sugerindo uma fraca validade discriminante dos itens que compõem esta dimensão.

Não obstante, a estrutura de seis dimensões revelou um bom ajustamento aos dados, assim como bons índices de fiabilidade (através do alfa de Cronbach e da Fiabilidade Compósita) e evidência de validade convergente (através da variância extraída da média) para a escala completa e para cada uma das seis dimensões que a compõem. No que diz respeito à avaliação da validade discriminante, não foi encontrada evidência para duas dimensões: Acordo nas práticas parentais e Suporte coparental; sendo estas responsáveis por sete das oito comparações em que não foi confirmada validade discriminante. Este resultado sugere que ambas as dimensões podem não ser precisas o suficiente para avaliar o conceito latente a que se referem, para além daquele que as restantes dimensões avaliam.

De forma semelhante, a análise fatorial confirmatória da versão breve da ERC revelou um bom ajustamento aos dados e também com os índices de fiabilidade e de validade convergente. Contudo, também na versão breve tiveram de ser eliminados os dois itens que compõem a dimensão Divisão de tarefas parentais, e ainda um dos itens que representam a dimensão Exposição ao conflito. Da mesma forma que na versão completa, também na breve foram corrigidas correlações entre os erros, nomeadamente entre os itens 25-27, 16-22, e 1-4. Tendo em conta os parâmetros avaliados neste estudo, pode concluir-se que há evidências de validade de construto da versão completa e da versão breve de ERC numa amostra comunitária e de baixo risco de pais/mães em Portugal, ainda que estudos futuros sejam necessários para corroborar os resultados encontrados no presente estudo.

Para avaliar a validade concorrente das versões completa e breve da ERC, foram realizadas correlações simples entre estas e as dimensões da Escala Revista de Ajustamento Diádico (RDAS) e do Questionário de Capacidades e Dificuldades (SDQ). No que diz respeito à validade concorrente com a relação diádica, foram encontradas correlações moderadas e na direção esperada entre as três dimensões de ajustamento diádico (Consenso, Coesão e Satisfação) e todas as dimensões de relação coparental e versão breve da ERC. Desta forma, foi encontrada evidência de validade concorrente da ERC quando comparada com uma escala que avalia a qualidade da relação diádica, uma outra componente importante do funcionamento familiar, ainda que concetualmente independente (Feinberg et al., 2012). Mais, as 
correlações encontradas entre as dimensões de ajustamento psicossocial das crianças (Problemas de Internalização, Problemas de Externalização, e Comportamentos Pró-sociais) e as dimensões de relação coparental e versão breve da ERC foram todas significativas e na direção esperada, ainda que globalmente mais fracas do que aquelas encontradas com o ajustamento diádico. Este resultado não é surpreendente, uma vez que há uma maior proximidade concetual entre a qualidade da relação diádica e a qualidade da relação coparental - que dizem ambas respeito à relação entre adultos -, do que entre a relação coparental e o ajustamento psicossocial das crianças. Ainda assim, foi encontrada evidência de validade concorrente entre a ERC e o SDQ, demonstrado uma associação significativa entre a qualidade da relação coparental e a existência de problemáticas emocionais, comportamentais, e sociais das crianças.

\section{Limitações}

Este estudo teve algumas limitações que devem consideradas na interpretação dos seus resultados. Quanto à amostra do estudo, as características dos pais/mães portugueses (a maioria dos participantes eram altamente qualificados, com rendimento médio a alto) e os procedimentos de recrutamento (online e por amostragem não probabilística) podem ter influenciado os resultados deste estudo, e por este motivo não serem generalizáveis à população de pais/mães portugueses. Mais, os questionários preenchidos por pais e mães foram avaliados no seu conjunto, ainda que pais e mães possam ter perceções diferentes da sua relação coparental. Estudos anteriores (Lamela et al., 2018) avaliaram as qualidades psicométricas da ERC numa amostra de mães, sugerindo-se assim que estudos futuros possam examinar a invariância da estrutura fatorial entre pais e mães. Do ponto de vista metodológico, algumas das dimensões da ERC revelaram fraca validade discriminante além de terem sido encontradas diversas correlações elevadas entre pares de erros da dimensão Suporte coparental, sugerindo que esta dimensão requer uma investigação teórica e metodológica mais detalhada sobre a sua especificidade face às restantes dimensões. Por fim, o presente estudo analisou apenas a estrutura fatorial, a consistência interna e a validade concorrente das versões completa e breve da ERC, e estudos futuros deverão avaliar a fiabilidade teste-reteste e a validade preditiva desta medida.

\section{Conclusão}

O presente estudo é um contributo no esforço comum dos investigadores portugueses para o desenvolvimento de medidas de avaliação da coparentalidade e igualmente de estudos de adaptação e de validação de instrumentos para a população portuguesa. De reforçar que este estudo tem singularidades que não foram tidas em consideração ou aplicadas em estudos anteriores, sendo que a sua aplicação foi pensada tanto para mães como para pais. Não se ficou apenas pela validação de uma das versões, mas sim tanto da escala completa como da breve, o que expande o conhecimento sobre a validade e confiabilidade de ambas as versões da escala. Estas especificidades reforçam a importância deste estudo, no entanto também é de salientar que alguns dados que foram aqui observados (e.g., a eliminação da Dimensão de tarefas) vão ao encontro do estudo original (Feinberg et al., 2012) e de estudos portugueses (Lamela et al., 2015; Lamela et al., 2018). 0 presente estudo dá resposta à insuficiência de instrumentos validados para a população portuguesa neste domínio e, sendo este o único instrumento que é sustentado por um modelo teórico de referência, os dados até aqui apresentados demonstram que o ERC aparenta ser uma medida potencialmente válida e confiável para incluir em futura investigação na área da coparentalidade.

\section{REFERÊNCIAS}

Abbass-Dick, J., Stern, S. B., Nelson, L. E., Watson, W., \& Dennis, C.-L. (2015). Coparenting breastfeeding support and exclusive breastfeeding: A randomized controlled trial. Pediatrics, 135(1), 102-110. https://doi.org/10.1542/peds.2014-1416

Abidin, R. R., \& Brunner, J. F. (1995). Development of a parenting alliance inventory. Journal of Clinical Child Psychology, 24(1), 31-40. https://doi.org/10.1207/s15374424jccp2401_4

Altenburger, L. E., Lang, S. N., Schoppe-Sullivan, S. J., Kamp Dush, C. M., \& Johnson, S. (2017). Toddlers' fifferential susceptibility to the effects of coparenting on social-emotional adjustment. International Journal of Behavioral Development, 41(2), 228-237. https://doi.org/10.1177/0165025415620058

Altenburger, L. E., Schoppe-Sullivan, S. J., Lang, S. N., Bower, D. J., \& Kamp Dush, C. M. (2014). Associations between prenatal coparenting behavior and observed coparenting behavior at 9-months postpartum. Journal of Family Psychology, 28(4), 495-504. https://doi.org/10.1037/fam0000012

Belsky, J., Crnic, K., \& Gable, S. (1995). The determinants of coparenting in families with toddler boys: Spousal differences and daily hassles. Child Development, 66(3), 629-642.

Busby, D. M., Christensen, C., Crane, D. R., \& Larson, J. (1995). A revision of the dyadic adjustment scale for use with distressed and nondistressed couples: construct hierarchy and multidimensional scales. 
Journal of Marital and Family Therapy, 21(3), 289-308. https://doi.org/10.1111/j.17520606.1995.tb00163.x

Cohen, J. (1988). Statistical power analysis for the behavioral sciences (2nd ed.). Lawrence Erlbaum Associates.

Costa, P. A., Pereira, H., \& Leal, I. (2011). Desenvolvimento da escala revista de ajustamento diádico (RDAS) com casais do mesmo sexo [Development of the Revised Dyadic Adjustment Scale (RDAS) with samesex couples]. Paper presented at the Actas do VIII Congresso Iberoamericano de Avaliação/Evaluación Psicológica, Lisboa, Portugal.

Costa, P. A., Tasker, F., Ramos, C., \& Leal, I. (2020). Psychometric properties of the parent's version of the SDQ and the PANAS-X in a community sample of Portuguese parents. Clinical Child Psychology and Psychiatry, 25(2), 520-532. https://doi.org/10.1177/1359104519891759

Dorsey, S., Forehand, R., \& Brody, G. (2007). Coparenting conflict and parenting behavior in economically disadvantaged single parent african american families: The role of maternal psychological distress. Journal of Family Violence, 22(7), 621-630. https://doi.org/10.1007/s10896-007-9114-y

Favez, N., Frascarolo, F., Lavanchy Scaiola, C., \& Corboz-Warnery, A. (2013). Prenatal representations of family in parents and coparental interactions as predictors of triadic interactions during infancy. Infant Mental Health Journal, 34(1), 25-36. https://doi.org/10.1002/imhj.21372

Favez, N., Tissot, H., Frascarolo, F., Stiefel, F., \& Despland, J.-N. (2016). Sense of competence and beliefs about parental roles in mothers and fathers as predictors of coparenting and child engagement in mother-father-infant triadic interactions. Infant and Child Development, 25(4), 283-301. https://doi.org/10.1002/icd.1934

Feinberg, M. E. (2002). Coparenting and the transition to parenthood: A framework for prevention. Clinical Child and Family Psychology Review, 5(3), 173-195. https://doi.org/10.1023/a:1019695015110

Feinberg, M. E. (2003). The internal structure and ecological context of coparenting: A framework for research and intervention. Parenting, Science and Practice, 3(2), 95-131. https://doi.org/10.1207/S15327922PAR0302_01

Feinberg, M. E., Brown, L. D., \& Kan, M. L. (2012). A multi-domain self-report measure of coparenting. Parenting, science and practice, 12(1), 1-21. https://doi.org/10.1080/15295192.2012.638870

Fleitlich, B., Loureiro, M., Fonseca, A., \& Gaspar, F. (2005). Questionário de Capacidades e Dificuldades (SDQ-Por). www.sdqinfo.com

Fornell, C., \& Larcker, D. F. (1981). Evaluating structural equation models with unobservable variables and measurement error. Journal of Marketing Research, 18(1), 39-50. https://doi.org/10.2307/3151312

Frizzo, G. B., Kreutz, C. M., Schmidt, C., Piccinini, C. A., \& Bosa, C. (2005). The concept of co-parenting: Implication for research and clinical practice. Journal of Human Growth and Development, 15(3), 84-93. https://doi.org/10.7322/jhgd.19774

Goodman, A., Lamping, D. L., \& Ploubidis, G. B. (2010). When to use broader internalising and externalising subscales instead of the hypothesised five subscales on the Strengths and Difficulties Questionnaire (SDQ): Data from British parents, teachers and children. Journal of Abnormal Child Psychology, 38(8), 1179-1191. https://doi.org/10.1007/s10802-010-9434-x

Goodman, R. (1997). The Strengths and Difficulties Questionnaire: A research note. Journal of Child Psychology and Psychiatry, 38(5), 581-586. https://doi.org/10.1111/j.1469-7610.1997.tb01545.x

Hair, J. F., Black, W. C., Babin, B. J., \& Anderson, R. E. (2009). Multivariate data analysis (7th ed.). Prentice Hall.

Hohmann-Marriott, B. (2011). Coparenting and father involvement in married and unmarried coresident couples. 73(1), 296-309. https://doi.org/10.1111/j.1741-3737.2010.00805.x

Holland, A. S., \& McElwain, N. L. (2013). Maternal and paternal perceptions of coparenting as a link between marital quality and the parent-toddler relationship. Journal of Family Psychology, 27(1), 117-126. https://doi.org/10.1037/a0031427

Jia, R., \& Schoppe-Sullivan, S. J. (2011). Relations between coparenting and father involvement in families with preschool-age children. Developmental Psychology, 47(1), 106-118. https://doi.org/10.1037/a0020802

Klasen, H., Woerner, W., Wolke, D., Meyer, R., Overmeyer, S., Kaschnitz, W., . . Goodman, R. (2000). Comparing the German Versions of the Strengths and Difficulties Questionnaire (SDQ-Deu) and the Child Behavior Checklist. European Child and Adolescent Psychiatry, 9(4), 271-276. https://doi.org/10.1007/s007870070030

Kline, P. (2000). The handbook of psychological testing (2nd ed.). Routledge. 
Lamela, D., Costa, R. N., \& Figueiredo, B. (2010). Modelos teóricos das relações coparentais: Revisão crítica. Psicologia em Estudo, 15(1), 205-216. https://doi.org/10.1590/S141373722010000100022

Lamela, D., Figueiredo, B., Bastos, A., \& Feinberg, M. (2015). Typologies of post-divorce coparenting and parental well-being, parenting quality and children's psychological adjustment. Child Psychiatry and Human Development, 47(5), 716-728. https://doi.org/10.1007/s10578-015-0604-5

Lamela, D., Morais, A., \& Jongenelen, I. (2018). Psychometric validation of the Coparenting Relationship Scale in Portuguese mothers. Avances en Psicología Latinoamericana, 36(3), 585-600. https://doi.org/10.12804/revistas.urosario.edu.co/apl/a.5564

Margolin, G., Gordis, E. B., \& John, R. S. (2001). Coparenting: A link between marital conflict and parenting in two-parent families. Journal of Family Psychology, 15(1), 3-21. https://doi.org/10.1037/08933200.15.1.3

Marôco, J. (2014). Análise de equações estruturais: Fundamentos teóricos, software e aplicações (2nd edition). Report Number.

McConnell, M. C., \& Kerig, P. K. (2002). Assessing coparenting in families of school-age children: Validation of the Coparenting and Family Rating System. Canadian Journal of Behavioural Science, 34(1), 4458. https://doi.org/10.1037/h0087154

McHale, J. P. (1995). Coparenting and triadic interactions during infancy: The roles of marital distress and child gender. Developmental Psychology, 31(6), 985-996. https://doi.org/10.1037/00121649.31.6.985

McHale, J. P., Kuersten-Hogan, R., \& Lauretti, A. (2001). Evaluating coparenting and family-level dynamics during infancy and early childhood: The coparenting and family rating system. In P. K. Kerig \& K. M. Lindahl (Eds.), Family observational coding systems (pp. 151-170). Erlbaum.

McHale, J. P., Kuersten-Hogan, R., \& Rao, N. (2004). Growing points for coparenting theory and research. Journal of Adult Development, 221-234. https://doi.org/10.1023/B:JADE.0000035629.29960.ed

Minuchin, S. (1974). Families and family therapy. Harvard University Press.

Pinto, T. M., Figueiredo, B., \& Feinberg, M. E. (2019). The Coparenting Relationship Scale-Father's Prenatal Version. Journal of Adult Development, 26(3), 201-208. https://doi.org/10.1007/s10804018-9308-y

Puhlman, D. J., \& Pasley, K. (2017). The maternal gatekeeping scale: Constructing a measure. Family Relations, 66(5), 824-838. https://doi.org/10.1111/fare.12287

Reader, J. M., Teti, D. M., \& Cleveland, M. J. (2017). Cognitions about infant sleep: Interparental differences, trajectories across the first year, and coparenting quality. Journal of Family Psychology, 31(4), 453463. https://doi.org/10.1037/fam0000283

Rothenberger, A., \& Woerner, W. (2004). Strengths and Difficulties Questionnaire (SDQ)--evaluations and applications. European Child \& Adolescent Psychiatry, 13 Suppl 2, Ii1-2. https://doi.org/10.1007/s00787-004-2001-7

Schoppe-Sullivan, S. J., \& Mangelsdorf, S. C. (2013). Parent characteristics and early coparenting behavior at the transition to parenthood. Social Development, 22(2), 363-383. https://doi.org/10.1111/sode.12014

Spanier, G. B. (1976). Measuring dyadic adjustment: New scales for assessing the quality of marriage and similar dyads. Journal of Marriage and Family, 38(1), 15-28. https://doi.org/10.2307/350547

Teubert, D., \& Pinquart, M. (2010). The association between coparenting and child adjustment: A metaanalysis. Parenting, 10(4), 286-307. https://doi.org/10.1080/15295192.2010.492040

Van Egeren, L. A., \& Hawkins, D. P. (2004). Coming to terms with coparenting: Implications of definition and measurement. Journal of Adult Development, 11(3), 165-178. https://doi.org/10.1023/B:JADE.0000035625.74672.0b

Warnick, E. M., Bracken, M. B., \& Kasl, S. (2008). Screening efficiency of the Child Behavior Checklist and Strengths and Difficulties Questionnaire: A systematic review. Child and Adolescent Mental Health, 13(3), 140-147. https://doi.org/10.1111/j.1475-3588.2007.00461.x

Historial do artigo

Recebido 09/2019

Aceite $\quad 06 / 2020$

Publicado 08/2020 